\title{
Erythrocyte Docosahexaenoic Acid Correlates with the Visual Response of Healthy, Term Infants
}

\author{
M. MAKRIDES, K. SIMMER, M. GOGGIN, AND R. A. GIBSON \\ Department of Paediatrics and Child Health [M.M., K.S., R.A.G.] and Department of Ophthalmology [M.G.]. \\ Flinders Medical Centre, Adelaide, South Australia 5042
}

\begin{abstract}
Recent studies have reported that formulafed preterm infants score lower on visual and developmental tests relative to breast-fed preterm infants. This phenomenon has been associated with the presence of docosahexaenoic acid (DHA), an omega-3 fatty acid, in breast milk and its absence from infant formula. To investigate the possibility that DHA status of healthy, term infants is also related to neuronal function of the visual pathway, we studied the erythrocyte fatty acid profiles of 16 infants at $22.3 \pm 3.9 \mathrm{wk}$ of age and related these to maturity of the visual pathway as assessed by visualevoked potentials. Healthy, term infants fed breast milk had better visual-evoked potential acuity $(p<0.05)$ and higher DHA levels $(p<0.001)$ than infants who received infant formula as their major energy source. There was a positive correlation between erythrocyte DHA and visualevoked potential acuity $(p<0.01)$. The data are preliminary and the long-term effects as yet unknown. However, our results suggest that there is an urgent need to evaluate the dietary fatty acid supply of formula-fed term infants. (Pediatr Res 34: 425-427, 1993)
\end{abstract}

\section{Abbreviations}

LC-PUFA, long-chain polyunsaturated fatty acid DHA, docosahexaenoic acid

AA, arachidonic acid

EFA, essential fatty acid

LA, linoleic acid

ALA, $\alpha$-linolenic acid

VEP, visual-evoked potential

MAR, minimum angle of resolution

$\log M A R, \log$ of the minimum angle of resolution.

The perceived role of the dietary fat supply in infancy has notably changed over the last decade. Dietary fat has been primarily considered an exchangeable energy source required for rapid growth. However, recent studies on the effects of LCPUFA, such as DHA and AA, suggest that the quality and composition of dietary fat may be vital for optimal growth and neuronal development during infancy.

Until the 1980 s, it was widely accepted that the two precursor EFA. LA (the omega 6 precursor) and ALA (the omega- 3 precursor), could be fully metabolized to AA and DHA, respectively,

Received for rapid publication November 5, 1992; accepted December 10, 1992. Correspondence: Maria Makrides, Dietitian, Ph.D. student, Department of Paediatrics and Child Health. Flinders Medical Centre. Bedford Park. Adelaide. South Australia 5042 .

Supported by Scotia Pharmaceuticals and Nestle Australia. and hence meet all the EFA requirements of infancy (1). Support for this belief was based on the fact that most infants receiving formulas containing only I A and A I A achieved adequate growth rates.

In contrast to formula, breast milk contains a full complement of omega-6 and omega-3 LC-PUFA including DHA and AA, which formula-fed infants must synthesize from precursors. In preterm infants, biochemical studies comparing breast and formula feeding indicate 8 to $10 \%$ less AA and 13 to $48 \%$ less DHA in the erythrocyte lipids of formula-fed infants (2). We have reported similar differences in term infants (2), suggesting that infant formulas containing only LA and ALA may not be effective at meeting the full EFA requirements of healthy infants.

These biochemical changes are clinically relevant, as studies have shown that dietary fatty acid supply affects physiologic function. Preterm formula-fed infants have a measurable reduction in neuronal function of the visual pathway, as measured by electroretinogram (3), VEP, and behavioral measures of visual acuity (4) relative to preterm breast-fed infants. These physiologic parameters were related to the DHA status of these infants and corrected by the addition of DHA containing fish oil to infant formula $(3,4)$.

DHA and AA form a large proportion of total lipids in the brain and retina and are primarily accreted into the brain during the "brain growth spurt," which begins in the last trimester of pregnancy and continues through early postnatal life (5). Previous research has focused on the preterm infant as being at greatest risk of disturbed fat accretion.

Our primary aim was to investigate whether the dietary supply of these fatty acids is as important to term infants as it is to preterm infants. To test the hypothesis that DHA status is related to the maturity of the visual pathway of healthy, term infants, we studied the fatty acid profiles of infants fed breast milk and infant formula and related these to their VEP. The VEP is the summed cortical response to a temporal change of a visual stimulus and is often used to assess the visual function of preverbal children. We chose the VEP as our assessment of the maturity of the visual pathway because few other noninvasive. reliable, and reproducible tests were available and studies indicate that VEP acuity is used as a measure of neuronal maturation of this pathway (6).

\section{MATERIALS AND METHODS}

Subjects. Healthy infants who were approximately 5 mo of age and born at term with appropriate weight for gestational age were enrolled into the study from immunization and postnatal (Child, Adolescent and Family Health Service) clinics. Of 22 infant-mother pairs asked to participate, four declined, one was subsequently excluded on the basis of the result of the ophthalmic examination, and one could not attend the stipulated clinic 
times, leaving 16 infants. Consent was obtained from the caregivers of these infants following guidelines approved by the Clinical Investigations (Ethics) Committee of Flinders Medical Centre, the Research Ethics Committee of the Adelaide Children's Hospital, and the Research Committee and the Ethics Sub-Committee of Child, Adolescent and Family Health Service. Consenting infant-mother pairs subsequently attended two hospital clinics within a week. On the first visit, mothers answered a short questionnaire detailing feeding patterns, socioeconomic status (7), and parity. The infants were weighed and measured, a blood sample was taken, and a complete ophthalmic examination was carried out. Infants with astigmatism or large refractive error were excluded from the study. On the second visit, a VEP was performed.

Diets. The formula-fed infants received one of three infant formulas, each of which had a similar fatty acid composition. Total saturates ranged from 48 to $50 \%$, monounsaturates from 34 to $36 \%$, LA from 12 to $15 \%$, and ALA from 1 to $1.6 \%$ of total fatty acids. The LA:ALA ratios were similar and ranged from 9.4 to 11.3 .

Both groups of infants were receiving solids, predominantly rice cereal and stewed fruit. None of the infants were receiving detectable quantities of DHA or AA from solids.

Fatty acid analysis of blood. One hundred fifty to $200 \mu \mathrm{L}$ of whole blood were obtained from a thumb prick and transferred to tubes containing lithium heparin. Plasma and erythrocytes were separated, and lipids were extracted, methylated, and quantified by capillary gas chromatography by following procedures previously reported (2).

$V E P$. Infants sat with their mothers, $1 \mathrm{~m}$ away from a monitor that presented high-contrast black-and-white checkerboard-pattern stimuli. A constant reversal rate of $2 / \mathrm{s}$ was used to elicit responses to checks with squares subtending visual angles of 7 . $17,34,69$, and $137 \mathrm{~min}$ of arc. Responses were obtained from an active electrode placed $3 \mathrm{~cm}$ above the inion, a reference electrode in a midfrontal position and an inactive electrode on the forehead. Recording occurred when infants were alert and their gaze directed at the monitor. A bell was used to help maintain infants' attention. Three recordings were taken at each check size to ensure reproducibility.

Peak-to-peak amplitude was determined for each check size. The amplitude of the evoked potential diminishes as check size decreases. Extrapolation of a regression line fitting VEP amplitude to $\log$ (check size) through $0-\mu \mathrm{V}$ amplitude gives the smallest check size that, if tested, would render a positive wave (6). This point is defined as the logMAR or VEP acuity.

\section{RESULTS}

Of the 16 infants who successfully completed the study, eight were exclusively breast-fed and eight received $>70 \%$ of their nutrition from infant formula. All infants were from families with similar social class (Table 1). There was no difference in age between the two groups, and breast-fed infants had a significantly smaller MAR (i.e. better VEP acuity) than those who had been predominantly formula-fed (Tabie 1). There was no correlation between postnatal age and VEP acuity, but the range of values reported for VEP acuity was within the range reported for healthy, term infants at 5 mo of age (6).

Infants fed breast milk had a greater proportion of erythrocyte DHA and less erythrocyte LA relative to those who had received infant formula as their major energy source (Table 1). There was a significant correlation between logMAR (VEP acuity) and the proportion of DHA $(r=-0.65, p<0.01)$ and LA $(r=+0.66, p$ $<0.01$ ) in erythrocyte phospholipids (Fig. 1).

\section{DISCUSSION}

This is the first report of a positive correlation between VEP acuity of healthy, term infants and the level of erythrocyte DHA.
Table 1. VEP acuity and erythrocyte phospholipid DHA and LA in breast-fed and predominantly formula-fed infants

\begin{tabular}{lcc}
\multicolumn{1}{c}{ Parameter } & $\begin{array}{c}\text { Breast-fed } \\
\text { infants } \\
(n=8)\end{array}$ & $\begin{array}{c}\text { Formula-fed } \\
\text { infants } \\
(n=8)\end{array}$ \\
\hline Age (wk \pm SD) & $22.4 \pm 3.7$ & $22.3 \pm 4.3$ \\
Parity (no. children \pm SD) & $1.7 \pm 0.7$ & $1.5 \pm 0.7$ \\
Social status (social score \pm SD) & $5.1 \pm 0.6$ & $5.3 \pm 1.2$ \\
VEP acuity (logMAR \pm SD) & $0.42 \pm 0.29$ & $0.74 \pm 0.27^{*}$ \\
Erythrocyte DHA $(\% \pm$ SD) $\dagger$ & $6.16 \pm 0.98$ & $3.31 \pm 0.82 \ddagger$ \\
Erythrocyte LA $(\% \pm$ SD) $\dagger$ & $9.56 \pm 1.15$ & $12.58 \pm 1.02 \ddagger$
\end{tabular}

$$
\begin{aligned}
& { }^{*} p<0.05 . \\
& +\% \text { of total fatty acid methyl esters. } \\
& \ddagger p<0.0001 \text {. }
\end{aligned}
$$
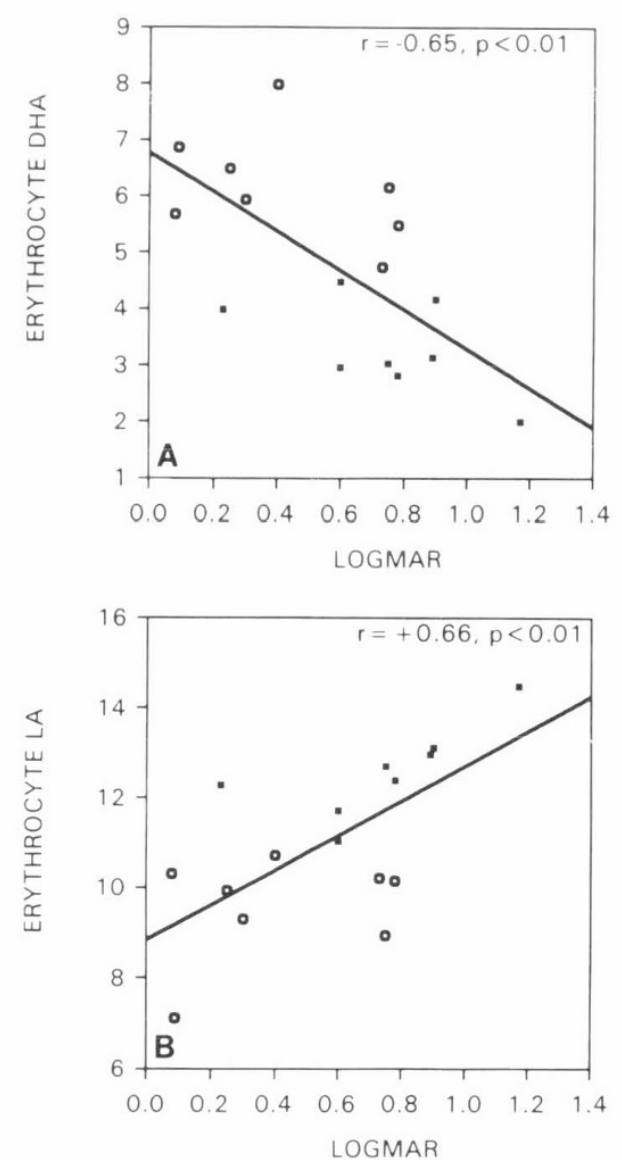

Fig. 1. A, Relationship between erythrocyte phospholipid DHA and $\log$ MAR. B, Relationship between erythrocyte phospholipid LA and $\operatorname{logMAR}$. O, infants exclusively fed breast milk; $\mathbf{E}$, infants predominantly fed infant formula. High logMAR indicates poor visual acuity, and lower values imply better acuity. $\operatorname{LogMAR} 0=20 / 20$ Snellen equivalent, and $\log$ MAR $1=20 / 200$ Snellen equivalent.

This suggests that term infants may be equally susceptible to altered visual/neuronal function as preterm infants (3) if there is a suboptimal dietary supply of DHA. In support of this, Birch et al. (4) have recently demonstrated that breast-fed term infants have better VEP and forced-choice preferential-looking acuities relative to those fed infant formula, although the quantification of fatty acid status was not performed in these infants. The longterm effect of the differences that we and others report in VEP acuity between breast- and bottle-fed infants is unknown.

In addition to containing virtually no DHA, most infant formulas contain large amounts of LA. Our data suggest that 
these high levels of LA may inhibit the incorporation of DHA into erythrocyte phospholipids, because it is the higher levels of erythrocyte DHA that are associated with lower levels of erythrocyte LA. Indeed, our work with adults demonstrates that the incorporation of omega-3 LC-PUFA into erythrocytes is inhibited by diets rich in LA (8). This raises some questions regarding the nutritional adequacy of the dietary fatty acid supply of formula-fed infants. Should we add DHA to formula, or should we look for ways to correct the balance of LA to ALA and hence optimize metabolism of ALA to DHA? The inverse relationship between erythrocyte LA levels and VEP acuity suggests that this issue is important. Our previous work has shown that decreasing the LA:ALA ratio of formula from 20:1 to between $3: 1$ and $4: 1$ results in a significant improvement in the level of DHA incorporated into erythrocytes (2). It remains to be tested whether further manipulation of the LA:ALA ratio of infant formula will result in DHA levels and physiologic responses equivalent to those of breast-fed infants.

During the first 6 mo of life, there is a rapid maturation of the retinocortical sensory element of the human visual system that is reflected by an equally rapid improvement in VEP acuity (6). Studies tracking the VEP development of healthy, term infants between 2 and 7 mo of age indicate that there is an average improvement of $20 \%$ per month in VEP acuity, with adult levels being reached by 7 mo of age (6). However, these studies also indicate that at any given point in time there may be a substantial deviation from the mean VEP acuity. Our results, as well as those reported by Birch et al. (4), suggest that this variation may be explained by differences in LC-PUFA status of the infant. which in turn is affected by the dietary supply of EFA.

Large, long-term studies investigating the effect of dietary supply of EFA to term infants on subsequent maturation of the visual pathway are urgently needed. Evidence to suggest that breast-fed infants have a long-term intelligence quotient advantage over infants who have been fed formula has been apparent in the literature for many years (9) and has been recently supported by the work of Lucas et al. (10). Although these last two studies do not relate their findings to fatty acid supply, it is attractive to postulate. from data published in the last 5 y that deficiency of LC-PUFA such as DHA is potentially a nutritional factor related to these observations. We must continue to investigate ways of providing appropriate fat in infant diets and assessing its effect on developmental outcomes. This will best be achieved with programs targeting the promotion of breast feeding and additional studies investigating the optimal fatty acid composition for infant formula.

Acknowledgment. The authors thank Paul Weston for technical assistance with VEP recording and the Flinders Medical Centre blood clinic for blood collection.

\section{REFERENCES}

1. Cuthbertson WFJ 1976 Essential fatty acid requirements in infancy. Am J Clin Nutr 29:559-568

2. Clark KJ, Makrides M, Neumann MA, Gibson RA 1992 Determination of the optimal ratio of linoleic acid to alpha-linolenic acid in infant formulas. J Pediatr 120:S151-S158

3. Uauy RD, Birch DG. Birch EE. Tyson JE. Hoffman DR 1990 Effect of dietary omiega-3 fattiy acids on rctinal function of very-low birth weight neonates. Pediatr Res 28:485-492

4. Birch EE, Birch DG. Hoffman DR, Uauy R 1992 Dietary essential fatty acid supply and visual acuity development. Invest Ophthalmol Visual Sci 33:3242-3253

5. Innis SM 1991 Essential fatty acids in growth and development. Prog Lipid Res 30:39-103

6. Sokol S 1978 Measurement of infant visual acuity from pattern reversal evoked potentials. Vision Res 18:33-39

7. Daniel A 1983 Power. Privilege. and Prestige. Occupations in Australia. Longman Cheshire. Melbourne

8. Cleland LG. James MJ. Neumann MA. D'Angelo M, Gibson RA 1992 Linoleate inhibits EPA incorporation from dietary fish oil supplements in human subjects. Am J Clin Nutr 55:395-399

9. Rodgers B 1978 Feeding in infancy and later ability and attainment: a longitudinal study. Dev Med Child Neurol 20:421-426

10. Lucas A. Morlev R. Cole TJ. Lister G. Lesson-Pavne C 1992 Breast milk and subsequent intelligence quotient in children born preterm. Lancet 339:261264 